Revista Brasileira de Meteorologia, v.25, n.2, 248 - 259, 2010

\title{
PASTURA NATURAL DE SALTO (URUGUAY): RELACIÓN CON LA VARIABILIDAD CLIMÁTICA Y ANÁLISIS DE CONTEXTOS FUTUROS DE CAMBIO CLIMÁTICO
}

\author{
MARÍA LAURA BETTOLLI ${ }^{1}$; MIGUEL ÁNGEL ALTAMIRANO DEL CARMEN²; GABRIELA CRUZ \\ BRASESCO $^{3}$; FREDERICO RUDORFF ${ }^{4}$; ARLEN MARTÍNEZ ORTIZ ${ }^{5}$; JACINTO ARROYO ${ }^{6}$ Y JORGE $^{2}$ \\ ARMOA $^{7}$ \\ ${ }^{1}$ Departamento de Ciencias de la Atmósfera y los Océanos, Universidad de Buenos Aires (CONICET), \\ Buenos Aires, Argentina. \\ ${ }^{2}$ Instituto Nacional de Ecología, México DF, México. \\ ${ }^{3}$ Facultad de Agronomía, Universidad de la República, Montevideo, Uruguay. \\ ${ }^{4}$ Instituto de Pesquisa e Prevenção em Desastres Naturais, Brasil. \\ ${ }^{5}$ Ministerio del Ambiente y los Recursos Naturales, Managua, Nicaragua. \\ ${ }^{6}$ Instituto Geofísico del Perú, Perú. \\ ${ }^{7}$ Universidad Nacional de Asunción, Asunción, Paraguay.
}

bettolli@at.fcen.uba.ar, maltamir@ine.gob.mx,gcruz@fagro.edu.uy,fmrudorff@yahoo.com.br, amartinez@marena.gob.ni, jarroyo@axil.igp.gob.pe, jarmoa@pol.una.py

Recibido Septiembre 2008 - Aceptado Octubre 2009

\begin{abstract}
RESUMEN
En este trabajo se evaluó objetivamente la relación entre la variabilidad del rendimiento de la pastura en el departamento de Salto (Uruguay) y la variabilidad climática. Se analizaron también las posibles implicancias del cambio climático futuro. Se utilizaron datos diarios y mensuales de la estación meteorológica Salto en el período 1961-1990 y un registro de datos experimentales de crecimiento de pastura en el período 1980-1994. Adicionalmente, se emplearon salidas diarias de reanálisis del NCEP y de los Modelos de Circulación General HadCM3 y CSIRO-Mk2 para los escenarios A2 y B2. Las correlaciones simples entre las variables climáticas y la pastura confirmaron en términos cuantitativos la sensibilidad de la pastura a la variabilidad climática, explicando entre el 20\% y 58\% de la varianza en los rendimientos. Las mayores asociaciones se encontraron en otoño (principalmente con las variables relacionadas con la precipitación) y verano. En invierno no se observaron asociaciones significativas. El efecto conjunto de las variables climáticas explicó entre el 36\% y $86 \%$ de la variabilidad en el crecimiento de la pastura. Para los escenarios estudiados, los incrementos proyectados en las temperaturas mínima y máxima permitirían reducir las probabilidades de ocurrencia de temperaturas por debajo del umbral mínimo considerado para la pastura $\left(10^{\circ} \mathrm{C}\right)$ y aumentar las probabilidades de estrés térmico durante el verano. La precipitación presentaría incrementos que podrían aumentar la incidencia de excedentes hídricos en invierno. Los incrementos proyectados para el verano no compensarían los aumentos de la demanda hídrica atmosférica por mayores temperaturas.
\end{abstract}

Palabras clave: variabilidad y cambio climático, crecimiento de la pastura, Salto

\begin{abstract}
NATURAL PASTURE IN SALTO (URUGUAY): RELATIONSHIP WITH CLIMATE VARIABILITY AND ANALYSIS IN THE CONTEXT OF FUTURE CLIMATE CHANGE.

This work aimed to evaluate the relationship between natural pasture yield and climate variability in the district of Salto (Uruguay), and to analyze the possible implications of future climate change. Daily and monthly data from Salto meteorological station for the period 1961-1990 were used together with experimental data of pasture yield for the period 1980-1994. Moreover, NCEP reanalyses of daily data as well as daily outputs from General Circulation Models HadCM3 and CSIRO-Mk2 were
\end{abstract}


analyzed. The simple correlations between the climatic variables and the pasture yield confirm in quantitative terms the sensitivity of pasture yield to climate variability, accounting for $20 \%$ up to $58 \%$ of the variance. The highest associations were found in fall (mainly with precipitation variables), followed by summer. No significant associations were found in winter. The joint effect of the climatic variables on yield could explain between $36 \%$ and $86 \%$ of the pasture variability. The increments projected for the minimum and maximum temperatures may, respectivaly, reduce the probabilities of occurrences of temperatures below the minimum threshold considered for pasture $\left(10^{\circ} \mathrm{C}\right)$ and increase the probabilities of thermal stress, mainly in summer, respectively. Modeled increments in precipitation may raise the occurrences of precipitation excess during winter. On the other hand, the modeled precipitation increments during summer would not be enough to compensate the high water demand caused by the greater temperatures.

Keywords: climate variability and change, pasture growth, Salto

\section{INTRODUCCIÓN}

En Uruguay el $71 \%$ de la superficie está constituida por campo natural (Domínguez y Prieto, 2002; MGAP, 2002). Por campo natural se entiende una superficie cuya cobertura consiste en vegetación espontánea, principalmente de tipo herbáceo y mayoritariamente autóctona, asociada a la presencia de montes nativos y vegetación arbustiva. La riqueza de especies, así como la predominancia de algunas especies forrajeras sobre otras, responde no sólo a la región del país (interacciones clima - suelo) sino también al manejo que se realice del pastoreo (Chebataroff, 1960, Altesor et al., 1998). La ganadería involucra el $82 \%$ de los establecimientos agropecuarios y emplea a la mitad de la población rural activa (MGAP, 2002).

La región de interés se localiza en el Departamento de Salto, situado sobre el litoral noroccidental de Uruguay que ocupa una superficie de $14163 \mathrm{~km}$ cuadrados (Figura 1). La pastura natural de esta región está conformada por una mezcla de especies de distintos hábitos de crecimiento, ciclos (estivales e invernales), tipos fotosintéticos (C3 y C4), valor forrajero y requerimientos bioclimáticos. No existe una base de datos para los requerimientos de cada una de las especies nativas, sin embargo, los pastos de zonas templadas están constituidos por una mezcla de especies C3 y C4 que poseen requerimientos térmicos similares dentro de cada grupo.

El cambio climático actual y la recurrencia de fenómenos climáticos extremos, tales como sequías e inundaciones, tienen impactos en los sistemas humanos en forma directa y a través de las modificaciones que generan en los sistemas agrícolas y pastoriles en forma indirecta (Baethgen, 1997). Numerosos estudios documentan la variabilidad climática en esta región durante el siglo XX. La precipitación ha mostrado variabilidades en escalas que van desde la diaria a la multidecádica (Bidegain y Caffera, 1991; Barros et al., 2005). Las temperaturas (medias y extremas) también han mostrado variabilidad en distintas escalas e incremento en las últimas décadas (Bidegain y Krecl, 1998; Rusticucci y Renom, 2008).

El Informe Especial sobre Escenarios de Emisiones (SRES scenarios, por sus siglas en inglés) brinda proyecciones sobre las emisiones de gases de efecto invernadero para el siglo

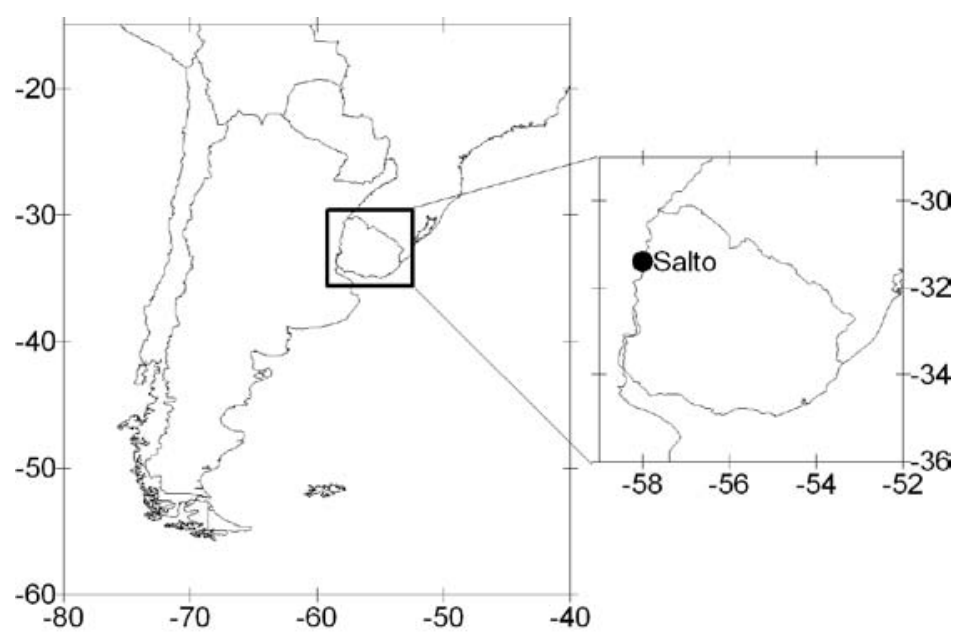

Figura 1 - Ubicación de la localidad de estudio. 
21 (Nakicenovic et al., 2000). Los escenarios “A” describen un mundo futuro con alto crecimiento económico, mientras que en los "B" ese crecimiento es más moderado. Los escenarios A1 y B1 suponen que habrá una globalización tal que las economías convergerán en su desarrollo. En los A2 y B2, se considera que el desarrollo se dará más a nivel regional (IPCC, 2001). Los resultados de los escenarios climáticos futuros obtenidos con los Modelos de Circulación General (MCG) indican para la región de interés una tendencia al aumento de la precipitación para los escenarios A2 y B2, en el segundo caso ligeramente inferior al primero (Barros et al., 2005; IPCC 2007). Para la temperatura, se proyecta un calentamiento especialmente significativo en el escenario A2 (Barros et al., 2005; IPCC 2007). Este calentamiento puede ser mayor en las regiones más continentales (Artigas y Salto) y en el caso más extremo llegaría a los $2,5^{\circ} \mathrm{C}$ para la década del 2050 para dicho escenario (Caffera et al., 2005).

En Uruguay, no se han encontrado trabajos de investigación donde se cuantifique objetivamente el efecto de la variabilidad climática sobre el rendimiento de la pastura natural en la región de interés a través de modelos estadísticos. Esto es debido probablemente a la falta de registros (particularmente del crecimiento de la pastura) lo suficientemente extensos en espacio y tiempo, que son necesarios para este tipo de estudios. Por otro lado, existen estudios sobre los posibles efectos del cambio climático sobre cultivos y pasturas implantadas (Giménez, 2006) y algunos sobre los efectos en el campo natural (Chiara y Cruz, 1997; Panario y Bidegain 1997).

Los sistemas de producción agropecuarios de Uruguay se ubican en una de las regiones más importantes de producción de alimentos del mundo. Por ello es importante cuantificar el efecto de la variabilidad del clima sobre dichos sistemas y considerar escenarios posibles de cambio climático. En este contexto, este trabajo representa un caso de estudio en el departamento de Salto (Uruguay) y se plantea como paso inicial en los estudios necesarios para evaluar la vulnerabilidad frente a la variabilidad y cambio climático de los sistemas pastoriles de la región.

Los objetivos de este trabajo fueron: diagnosticar y cuantificar objetivamente la relación entre las variables climáticas y el rendimiento de la pastura en Salto para distintos tipos de suelo y estaciones del año. Adicionalmente se realizaron proyecciones climáticas para la región con el fin de analizar posibles contextos climáticos futuros para el crecimiento de la pastura.

Esta investigación se desarrolló en el marco del estímulo por parte del Instituto Interamericano para la Investigación en Cambio Global (IAI) a la formación y consolidación de redes multinacionales y multidisciplinarias de investigación, sobre vulnerabilidad asociada a la variabilidad y al cambio climático.

\section{DATOS Y METODOLOGÍA}

\subsection{Datos}

Se utilizaron datos de rendimiento estacional de la pastura que corresponden a la tasa de crecimiento diario (kg MS ha-1 día-1; donde MS se refiere a masa seca) sobre la unidad cartográfica denominada Queguay Chico. Los suelos de dicha Unidad se caracterizan por tener como material generador el Basalto y presentar un relieve de lomadas fuertes y colinas con escarpas, así como rocosidad moderada (MGAP, 2005). Se utilizó información de rendimiento de la pastura creciendo sobre suelos de la referida Unidad, categorizados según Berreta (1998) como suelos superficiales pardo rojizos, superficiales negros y profundos, durante el período 1980-1994. Es importante destacar aquí que si bien el período analizado es de 15 años, es la única base de información oficial de la tasa de crecimiento de la pastura disponible, confiable y registrada en forma continua y homogénea a lo largo de los años.

Los datos climáticos utilizados incluyen datos diarios de precipitación y temperatura máxima (Tmáx) y mínima (Tmín) y promedios mensuales de temperatura media, velocidad del viento a 2 metros, heliofanía y humedad de la estación meteorológica de Salto (31.16 $\mathrm{S}$ y $57.53^{\circ} \mathrm{O}$, Figura 1 ) para el período 1961-1990, aportados por la Dirección Nacional de Meteorología de Uruguay (DNM).

Los reanálisis diarios del National Centers for Environmental Prediction (NCEP) en resolución espacial de $2.5^{\circ}$ x $2.5^{\circ}$ (lat x lon) fueron provistos por el NOAA-CIRES Climate Diagnostics Center en el período 1961-1990, considerado como período de referencia.

Adicionalmente, se usaron salidas diarias de los Modelos de Circulación General (MGC) HadCM3 (modelo del Hadley Centre-Reino Unido) en resolución espacial de $2.75^{\circ}$ x $3.75^{\circ}$ (lat $\mathrm{x}$ lon) y CSIRO-Mk2 (modelo del CSIRO Centre-Australia) en resolución espacial de $3.2^{\circ} \times 6.25^{\circ}$ (lat x lon). Estos modelos se encuentran entre los modelos disponibles que representan en forma más adecuada la circulación en el sudeste de Sudamérica (Barros et al., 2005). Las coordenadas utilizadas correspondieron al punto de enrejado más cercano a la estación meteorológica de Salto. Se consideraron los escenarios A2 y B2 (IPCC, 2001) y los horizontes temporales 2020, 2050 y 2080. Estos escenarios fueron seleccionados debido a que las condiciones de América Latina seguirán determinadas por el desarrollo económico, con uso de nuevas tecnologías "limpias", principalmente a nivel de regiones o localidades (Gay et al., 2006).

\subsection{Métodos}

\subsubsection{Caracterización Agroclimática de Referencia}


Para definir los rangos térmicos de crecimiento de las especies a utilizar se tomó como referencia los propuestos por Baradas (1994) (Tabla 1). Como la zona de estudio se ubica en una región de transición de las especies vegetales presentes (templadas y tropicales), se propone utilizar los umbrales de temperatura correspondientes a las categorías 2 y 3 de la Tabla 1. La categoría 2 involucra a las especies templadas (C3) que se han adaptado a condiciones de mayor temperatura y la categoría 3 alude a especies tropicales (C4) que se han adaptado a condiciones de menor temperatura. Las especies C3, presentan ciclo invernal y están productivas mayoritariamente en otoño, invierno y primavera. Las especies C4 presentan ciclo estival y permanecen productivas en primavera, verano y otoño. Las especies C4 son las más frecuentes, participando con $60 \%$ a 80\% de la cobertura del suelo (Berreta, 1998).

Los requerimientos hídricos del tapiz vegetal fueron estimados de acuerdo a la evapotranspiración potencial (ETP) mensual, calculada según Penman (FAO, 1979). Se elaboraron balances hídricos climáticos utilizando la metodología propuesta por Thornthwaite y Mather (1967) para cada tipo de suelo. La capacidad de almacenaje de agua se estimó de acuerdo a la profundidad, composición granulométrica y contenido de materia orgánica (Fernández, 1979). Para la estimación de ETP se utilizaron los promedios mensuales de temperatura media, velocidad del viento a $2 \mathrm{~m}$, heliofanía y humedad del período 1961-1990. La información de los suelos correspondió a: Brunosol Eutrico Típico LAc v, Perfil M13-04 (profundo); Litosol Eutrico Melanico Lac, Perfil M13-07 (superficial negro) y Litosol Eutrico Melanico FrMs, Perfil M13-06 (superficial rojo) (MGAP, 2005).

\subsubsection{Cuantificación de los Efectos de la Variabilidad Climática sobre el Crecimiento de la Pastura}

Para estudiar el efecto de la variabilidad climática sobre la pastura en el sitio de estudio, se calcularon los valores trimestrales de totales de precipitación, frecuencia de días de lluvia, frecuencia de días con lluvia inferior a la evapotranspiración potencial correspondiente a cada mes del trimestre. Para las temperaturas se calcularon valores medios de Tmáx y Tmín, frecuencia de días con Tmáx $>35^{\circ} \mathrm{C}$

a)
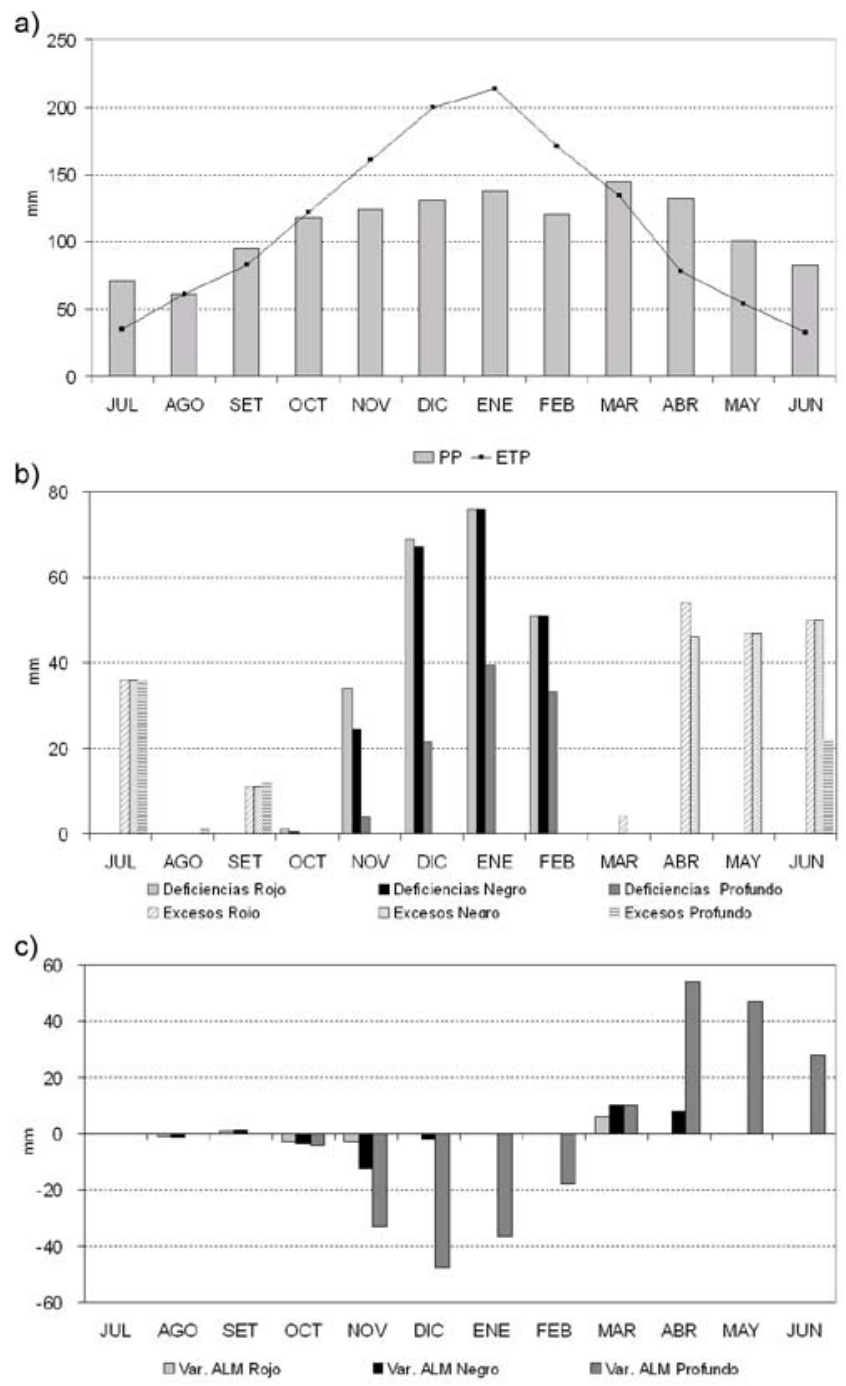

Figura 2 - Precipitación y evapotranspiración potencial promedio (a), balance hídrico climático de la pastura en cada tipo de suelo: deficiencias y excesos (b) y variación de almacenaje (c) para el período 1961-1990.

Tabla 1 - Rangos térmicos para los distintos grupos fotosintéticos, extractados de Baradas (1994)

\begin{tabular}{|c|c|c|c|}
\hline Categoría & $\begin{array}{c}\text { Grupo según patrón } \\
\text { fotosintético }\end{array}$ & $\begin{array}{c}\text { Rango T } \\
\text { sobrevivencia }\left({ }^{\circ} \mathbf{C}\right)\end{array}$ & $\begin{array}{c}\text { Rango T óptimo } \\
\left({ }^{\circ} \mathbf{C}\right)\end{array}$ \\
\hline $\mathbf{1}$ & $\mathrm{C}_{3}-$ adaptada a menores T & $5-30$ & $15-20$ \\
\hline $\mathbf{2}$ & $\mathrm{C}_{3}-$ adaptada a mayores T & $10-35$ & $25-30$ \\
\hline $\mathbf{3}$ & $\mathrm{C}_{4}-$ adaptada a menores T & $10-40$ & $15-30$ \\
\hline $\mathbf{4}$ & $\mathrm{C}_{4}-$ adaptada a mayores T & $15-45$ & $30-35$ \\
\hline
\end{tabular}


y frecuencia de días con $\mathrm{Tmín}<10^{\circ} \mathrm{C}$ correspondientes a los umbrales de temperatura de sobrevivencia de las categorías 2 y 3 de la Tabla 1 . Se agregó también al análisis la frecuencia de días con Tmáx $>30^{\circ} \mathrm{C}$ y frecuencia de días con Tmín $<5^{\circ} \mathrm{C}$. Los trimestres considerados correspondieron al verano (diciembreenero-febrero), otoño (marzo-abril-mayo), invierno (junio-julioagosto) y primavera (septiembre-octubre-noviembre).

La comparación de la variabilidad entre diferentes conjuntos de datos y/o estaciones del año se llevó a cabo con el coeficiente de variación, calculado como la división entre el desvío estándar y el valor medio de la variable en términos porcentuales (Devore, 2005).

La asociación entre las variables climáticas y la tasa de crecimiento diaria de la pastura fue estudiada en base al cálculo del coeficiente de correlación de primer momento de Pearson. La significancia estadística del coeficiente de correlación se testó con la distribución t-Student con N-2 grados de libertad (Devore, 2005). Para encontrar un conjunto de variables climáticas predictoras y estimar el efecto combinado de ellas sobre la tasa de crecimiento de la pastura, se utilizó un modelo de regresión múltiple de a pasos -stepwise multilinear regression- (Draper y Smith, 1981). Los grados de libertad y la significancia de la regresión dependen del número de variables predictoras y de la longitud de las series. Teniendo en cuenta esta limitación estadística se realizaron distintos ajustes con diferentes grupos de variables de entrada, a fin de analizar las respuestas y sensibilidad de los modelos.

\subsubsection{Contextos Climáticos Futuros}

La reducción de escala estadística se realizó utilizando el software Statistical DownScaling Model 3.1 (SDSM) (Wilby y Dawson, 2004) para construir los escenarios de cambio climático a escala local. El SDSM es de acceso libre a través del sitio web https://co-public.lboro.ac.uk/cocwd/SDSM/. En un primer paso, en cada trimestre se identificaron los conjuntos de variables predictoras para cada uno de los predictandos (precipitación y temperaturas máxima y mínima) en base a un análisis de correlaciones. En un segundo paso, se realizó el proceso de calibración utilizando regresiones múltiples en base a los predictores seleccionados. Estos modelos calibrados se verificaron utilizando generadores de tiempo, utilizando técnicas estocásticas para estabilizar la varianza de las series de tiempo diarias con el fin de obtener una mayor armonía con las observaciones. El modelo se calibró con las salidas de los reanálisis del NCEP en el período 1961-1975 y se validó para el período 1976-1990. En un tercer paso, se generaron los ensambles de las series diarias sintéticas para los escenarios de cambio climático antes mencionados utilizando los modelos calibrados en forma continua.
El estudio de variabilidad climática y su relación con la pastura se realizó sobre la base de los 15 años de registro de la pastura. La caracterización agroclimática de referencia y el análisis de contextos climáticos futuros se llevó a cabo utilizando un período de 30 años (1961-1990).

\section{RESULTADOS}

\subsection{Caracterización Agroclimática de Referencia}

La relación promedio entre precipitación y evapotranspiración potencial (ETP) para el período 1961-1990 mostró deficiencia de agua desde fines de primavera y durante todo el verano, mientras que los excedentes se presentaron de marzo a septiembre (Figura 2a). Los balances hídricos mostraron resultados ligeramente diferentes a los anteriores, debido a la capacidad amortiguadora de los suelos, en particular del suelo profundo. A mayor capacidad de almacenaje de agua del suelo, menores resultaron los períodos y magnitudes de deficiencia y exceso (Figuras 2b y 2c). Esto es debido a los mayores valores de variación de almacenaje en suelos profundos, tanto durante la etapa de extracción de agua del suelo (variación de almacenaje negativa) como durante la recarga del mismo (variación de almacenaje positiva) (Figura 2c). La componente ETP mostró una marcada variación estacional que explicaría
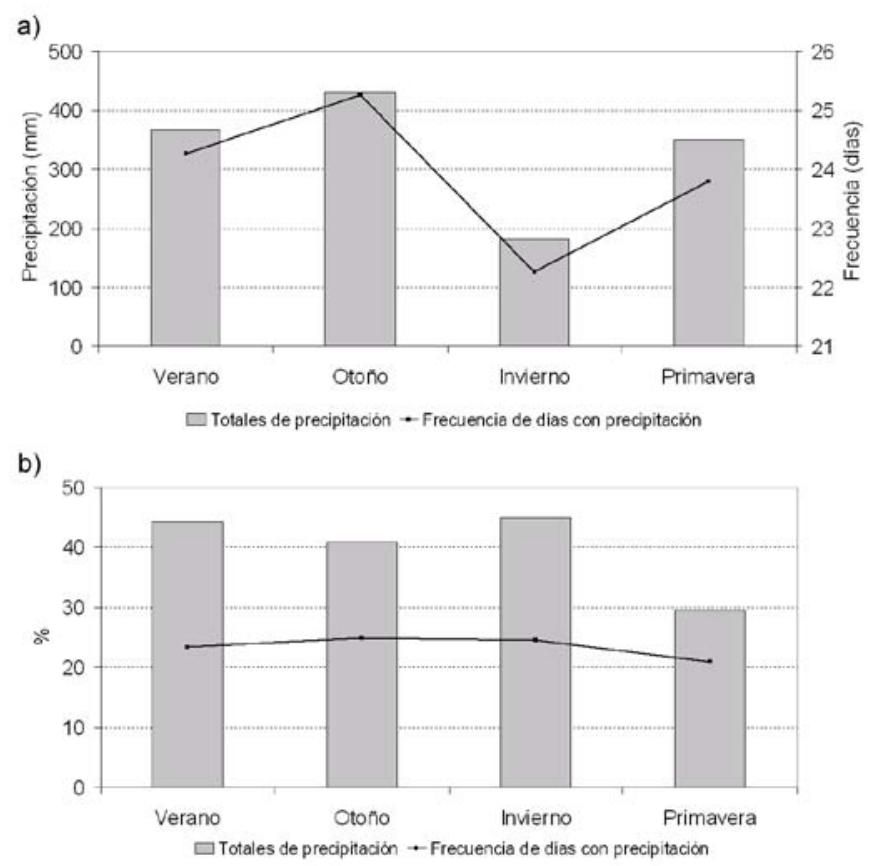

Figura 3 - Valores medios de precipitación por estación y frecuencias de días de lluvia (a) y sus respectivos coeficientes de variación (b) en el período 1980-1994. 
la estacionalidad promedio de deficiencias y excesos de los balances hídricos.

En el período coincidente con los datos de pastura (1980-1994), la región recibió en promedio 1330 mm de precipitación al año, distribuidos con un máximo en otoño y un mínimo principal en invierno (Figura 3a). La frecuencia de días con precipitación acompañó a los totales de precipitación. Durante los trimestres del verano, otoño e invierno, los totales de precipitación presentaron una variabilidad interanual superior al $40 \%$ (Figura $3 \mathrm{~b}$ ), lo que sugiere limitaciones cuando se caracteriza la región solamente en términos del promedio climático. Este comportamiento estuvo acompañado por la variación en la frecuencia de días de lluvia aunque con valores del orden del 24\% (Figura 3b).

La frecuencia estacional promedio de días con temperaturas superiores o inferiores respecto a los umbrales de sobrevivencia de la pastura (categorías 2 y 3 de Tabla 1) para el período 1980-1994 se muestra en la Figura 4. En invierno se presentaron temperaturas mínimas inferiores a $10^{\circ} \mathrm{C}$ en el $59 \%$ de los días. Durante el verano, algo más del 20\% de los días superaron el umbral de $35^{\circ} \mathrm{C}$ y sólo en 24 días de todo el registro de verano se superaron los $40^{\circ} \mathrm{C}$. El máximo de días con temperatura superior a $40^{\circ} \mathrm{C}$ correspondió al verano de 1989 (8 días), año de intensa sequía. Considerando que las especies que predominan en verano son las tropicales, cuyo umbral térmico máximo es de $40^{\circ} \mathrm{C}$, no existirían riesgos importantes de sobrevivencia vegetal por altas temperaturas. En invierno, en cambio, la elevada probabilidad de ocurrencia de temperaturas por debajo del umbral de tolerancia en ambos grupos vegetales, indican condiciones de riesgo.

La evolución trimestral de la tasa de crecimiento diario de la pastura presentó un marcado ciclo estacional, observándose las mayores tasas de crecimiento en verano y primavera (Figura 5a). El invierno presenta un valor mínimo de crecimiento de la pastura, en concordancia con las bajas temperaturas de la estación. En todos los trimestres la mayor tasa de crecimiento se presentó en el suelo profundo, siguiendo en importancia el suelo superficial negro y luego el rojo, en correspondencia con la capacidad de suministro de agua por parte de cada tipo de suelo. La variabilidad interanual de la tasa de crecimiento medida en términos del coeficiente de variación, presentó elevados valores para las cuatro estaciones del año (entre 30 y 50\%), siendo máxima sobre suelo superficial rojo (Figura 5b).

\subsection{Cuantificación de los Efectos de la Variabilidad Climática sobre el Crecimiento de la Pastura}

\subsubsection{Correlaciones Simples}

Los valores de los coeficientes de correlación entre las variables climáticas y las tasas de crecimiento de la pastura dependieron de la estación considerada y del tipo de suelo(Tabla 2).

El otoño presentó la mayor cantidad de coeficientes de correlación significativos al 95\% de confianza, especialmente en las variables indicadoras de humedad (Tabla 2). Se observó una relación directa entre la tasa de crecimiento y los montos totales de precipitación durante esta estación así como también con la frecuencia de días de lluvia. Al mismo tiempo, se observó una relación inversa con las frecuencias de días con precipitación que no superaron la ETP para la época. Se observó el efecto de las bajas temperaturas a través de las correlaciones significativas al $90 \%$ de confianza con la Tmín en el suelo superficial negro y con la frecuencia de días con Tmín inferior a los $10^{\circ} \mathrm{C}$ en el suelo superficial rojo (Tabla 2).

Durante el verano, las correlaciones significativas se presentaron con las variables de humedad para los tres tipos

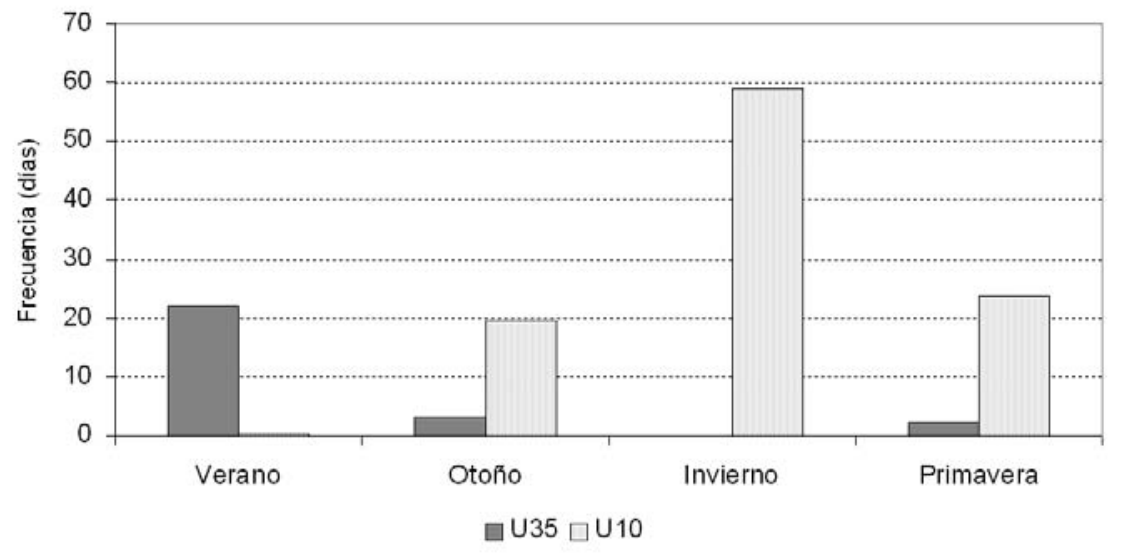

Figura 4 - Frecuencia estacional promedio de días con temperatura máxima superior a $35^{\circ} \mathrm{C}$ (U35) y frecuencia estacional promedio de días con temperatura mínima a $10^{\circ} \mathrm{C}$ (U10) en el período 1980-1994. 
a)

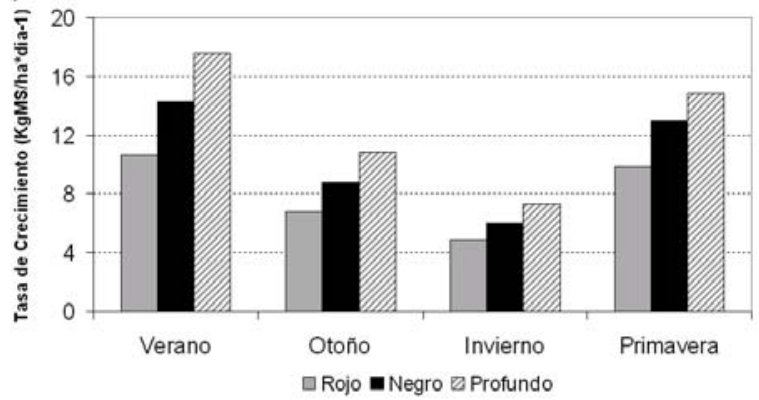

b)

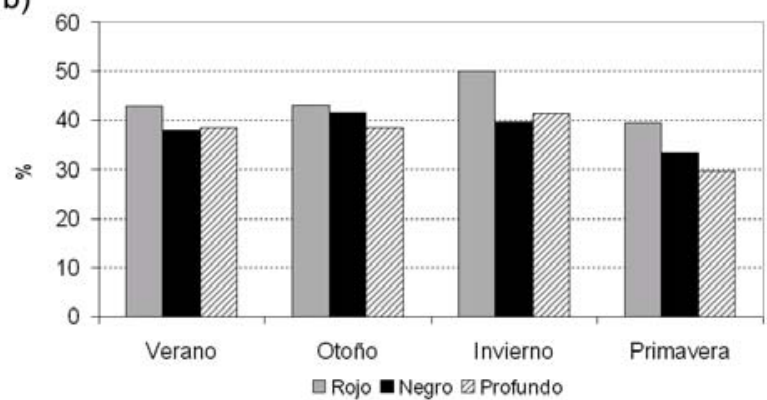

Figura 5 - Tasa promedio de crecimiento diario de la pastura (a) y coeficiente de variación (b) por estación para los suelos superficiales rojo y negro y profundo en el período 1980-1994.

Tabla 2 - Correlaciones entre las tasas de crecimiento diario de la pastura sobre los distintos suelos y las variables climáticas por estación. * (**) correlaciones significativas al 5\% (10\%), (PP Total: precipitación estacional; Frec PP: Frecuencia de días con precipitación; Frec PP<etp: Frecuencia de días con precipitación inferior a la evapotrasnpiración potencial; Tmáx: Temperatura máxima promedio; Tmín: temperatura mínima promedio; U35/U30: Frecuencia de días con temperatura máxima superior a $35^{\circ} \mathrm{C} / 30^{\circ} \mathrm{C}$; U10/U5: Frecuencia de días con temperatura mínima inferior a $10^{\circ} \mathrm{C} / 5^{\circ} \mathrm{C}$; Frec Heladas: Frecuencia de días con heladas agronómicas.)

\begin{tabular}{|c|c|c|c|c|c|c|c|}
\hline \multicolumn{4}{|c|}{ Verano } & \multicolumn{4}{|c|}{ Otoño } \\
\hline & Rojo & Negro & Profundo & & Rojo & Negro & Profundo \\
\hline PP Total & $0.49 * *$ & $0.45 * *$ & 0.60 * & PP Total & $0.55^{*}$ & $0.68 *$ & 0.69 * \\
\hline Frec PP & 0.60 * & $0.54 *$ & $0.58 *$ & Frec PP & $0.63 *$ & $0.71 *$ & $0.59 *$ \\
\hline Frec $P P<$ etp & $-0.51^{* *}$ & $-0.47 * *$ & $-0.72 *$ & Frec PP<etp & $-0.69 *$ & $-0.76^{*}$ & $-0.68 *$ \\
\hline Tmáx & -0.21 & -0.36 & -0.36 & \begin{tabular}{|l} 
Tmáx \\
\end{tabular} & -0.16 & -0.11 & -0.16 \\
\hline Tmín & 0.04 & -0.25 & -0.03 & Tmín & 0.42 & $0.47 * *$ & 0.36 \\
\hline U35 & -0.34 & $-0.49 * *$ & $-0.53 *$ & U35 & -0.28 & -0.29 & -0.07 \\
\hline U30 & -0.11 & -0.24 & -0.16 & U30 & -0.24 & -0.16 & -0.21 \\
\hline U10 & -0.16 & -0.01 & -0.08 & U10 & $-0.45 * *$ & -0.40 & -0.22 \\
\hline U5 & & & & U5 & -0.25 & -0.23 & -0.08 \\
\hline Frec Heladas & & & & Frec Heladas & -0.35 & -0.28 & -0.30 \\
\hline \multicolumn{4}{|c|}{ Invierno } & \multicolumn{4}{|c|}{ Primavera } \\
\hline & Rojo & Negro & Profundo & & Rojo & Negro & Profundo \\
\hline PP Total & 0.08 & 0.22 & 0.15 & PP Total & 0.08 & 0.05 & 0.17 \\
\hline Frec PP & 0.24 & 0.44 & 0.37 & Frec PP & 0.34 & 0.28 & 0.27 \\
\hline Frec $P P<$ etp & -0.13 & -0.40 & -0.42 & Frec PP<etp & -0.42 & -0.26 & -0.14 \\
\hline Tmáx & 0.12 & 0.04 & 0.21 & Tmáx & -0.02 & 0.05 & $0.49 * *$ \\
\hline Tmín & 0.31 & 0.32 & -0.06 & Tmín & 0.25 & 0.29 & $0.62 *$ \\
\hline U35 & & & & U35 & 0.33 & 0.39 & 0.23 \\
\hline U30 & -0.27 & -0.40 & -0.35 & U30 & -0.23 & -0.27 & -0.18 \\
\hline U10 & -0.22 & -0.24 & 0.14 & U10 & -0.43 & $-0.46 * *$ & $-0.58 *$ \\
\hline U5 & -0.28 & -0.29 & 0.08 & U5 & 0.10 & 0.10 & -0.21 \\
\hline Frec Heladas & -0.34 & -0.38 & 0.02 & Frec Heladas & 0.13 & 0.02 & -0.15 \\
\hline
\end{tabular}

Tabla 3 - Variables climáticas estimadoras de la tasa de crecimiento de la pastura, resultantes de la regresión múltiple. Entre paréntesis se indica el signo del coeficiente de la regresión para cada variable. R2: coeficiente de determinación.

\begin{tabular}{|c|c|c|c|}
\hline \multicolumn{4}{|c|}{ Verano } \\
\hline Tipo de Suelo & Rojo & Negro & Profundo \\
\hline $\begin{array}{l}\text { Variables en la } \\
\text { ecuación }\end{array}$ & $(+)$ Frec PP & $\begin{array}{l}\text { (+)Frec PP } \\
\text { (-) Tmín } \\
(+) \text { U30 } \\
(+) \text { PP Total }\end{array}$ & $\begin{array}{l}\text { (-) Frec PP<etp } \\
(+) \text { U30 } \\
\text { (-) Tmín } \\
\text { (+) PP Total } \\
\text { (-) Tmáx }\end{array}$ \\
\hline $\mathbf{R}^{2}$ & 0.36 & 0.71 & 0.86 \\
\hline \multicolumn{4}{|c|}{ Otoño } \\
\hline Tipo de Suelo & Rojo & Negro & Profundo \\
\hline $\begin{array}{l}\text { Variables en la } \\
\text { ecuación }\end{array}$ & $\begin{array}{l}\text { (-) Frec PP<etp } \\
(+) \text { Tmín } \\
\text { (-) Tmáx }\end{array}$ & $\begin{array}{l}\text { (-) Frec PP<etp } \\
(+) \text { Tmín } \\
(+) \text { U5 }\end{array}$ & $\begin{array}{l}\text { (+)PP Total } \\
\text { (-)Frec Heladas } \\
(+) \text { U35 }\end{array}$ \\
\hline $\mathbf{R}^{2}$ & 0.57 & 0.72 & 0.69 \\
\hline \multicolumn{4}{|c|}{ Primavera } \\
\hline Tipo de Suelo & \begin{tabular}{|l|} 
Rojo \\
\end{tabular} & Negro & Profundo \\
\hline $\begin{array}{l}\text { Variables en la } \\
\text { ecuación }\end{array}$ & $\begin{array}{l}\text { (-) U10 } \\
\text { (-) Tmáx } \\
\text { (-) PP Total } \\
\text { (-) Frec PP<etp }\end{array}$ & $\begin{array}{l}\text { (-) U10 } \\
\text { (-) Tmáx } \\
\text { (-) PP Total }\end{array}$ & $\begin{array}{l}\text { (+) Tmín } \\
\text { (-)Frec PP }\end{array}$ \\
\hline $\mathbf{R}^{2}$ & 0.53 & 0.42 & 0.44 \\
\hline
\end{tabular}


de suelo (Tabla 2). En esta estación la pastura manifestó sensibilidad a las altas temperaturas, como se evidencia en las correlaciones significativamente negativas con la frecuencia de días con Tmáx superior a $35^{\circ} \mathrm{C}$ en los suelos superficial negro y profundo (Tabla 2). Sin embargo, también podría estar marcando una respuesta al estrés hídrico ya que mayores temperaturas implican mayores niveles de ETP. Un incremento de temperatura marca mayor demanda hídrica atmosférica que, en los suelos profundo y superficial negro, se cubre en gran medida con el agua almacenada en el perfil. El suelo superficial rojo, que no mostró resultados significativos en la correlación con la frecuencia de días con temperatura mayor a $35^{\circ} \mathrm{C}$, ya no cuenta con agua en el suelo a partir de diciembre (Figura 2c), lo que implica que aunque la temperatura aumente, no habrá respuesta en el crecimiento vegetal dado que no existe agua almacenada que pueda verse afectada.
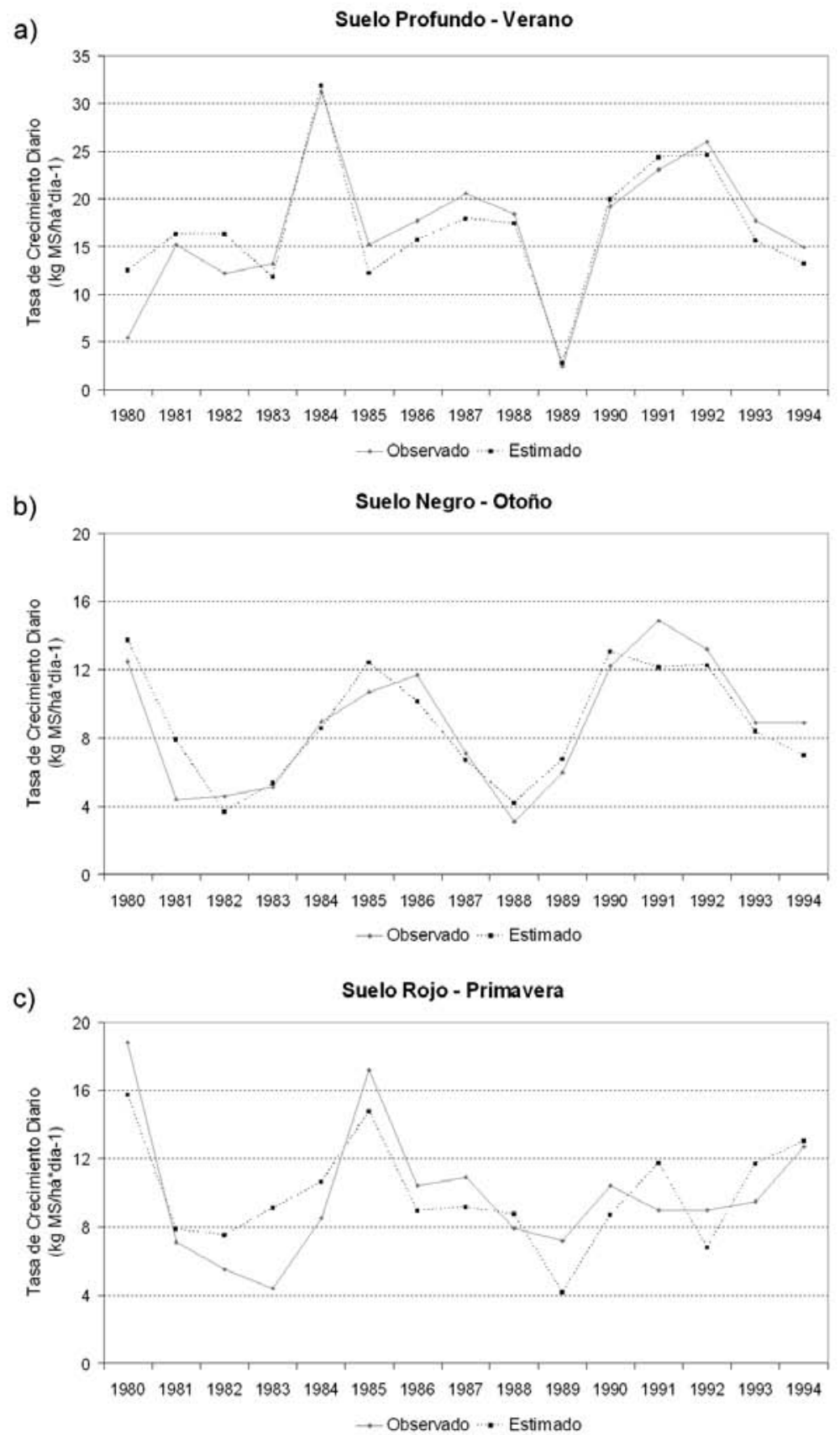

Figura 6 - Tasas observadas y estimadas de crecimiento de la pastura durante el período 1980-1994 para el verano en suelo profundo, otoño en suelo negro y primavera en suelo rojo. 
En la primavera no se encontraron correlaciones significativas con las variables indicadoras de humedad. Este resultado se debe a que el almacenaje de agua durante los inviernos estaría supliendo la necesidad hídrica de las primaveras, sobre todo en el suelo profundo (Figura 2c). El tipo de suelo sobre el cual se desarrolla la pastura condiciona la oferta hídrica para las plantas. Los suelos profundos poseen mayor capacidad de retención de agua y pueden contribuir con una mayor cantidad de la misma durante más tiempo que suelos superficiales, lo que se visualiza en la variación de almacenaje negativa que representa la extracción de agua del suelo (Figura 2c). Nótese que la correlación entre la tasa de crecimiento de la pastura y las variables indicadoras de humedad resultó mayor en el suelo rojo que en el negro y el profundo. Este resultado, aún sin presentar significancia estadística estaría evidenciando cierta tendencia. La pastura del suelo profundo mostró asociación directa con las temperaturas extremas y negativa con la frecuencia de días con Tmín inferior a $10^{\circ} \mathrm{C}$ (Tabla 2). En menor medida, las pasturas del suelo superficial negro mostraron sensibilidad a esta última variable (Tabla 2).

No se presentaron correlaciones significativas en invierno. Sin embargo, se observó cierta dependencia negativa, particularmente, con la frecuencia de heladas.

\subsubsection{Regresiones Múltiples}

Con el fin de estimar el efecto conjunto de las variables climáticas sobre el desarrollo de la pastura se utilizó un modelo de regresión múltiple setpwise. En la Tabla 3 se presenta un resumen de las regresiones obtenidas estadísticamente significativas al $5 \%$. Todas las estaciones del año, excepto el invierno donde no se encontraron regresiones significativas, presentaron un conjunto de variables climáticas a través de las cuales se puede estimar la tasa de crecimiento según el tipo de suelo. Las varianzas explicadas por los modelos variaron entre 0.36 y 0.86 (Tabla 3 ) cuantificando en alguna

a)

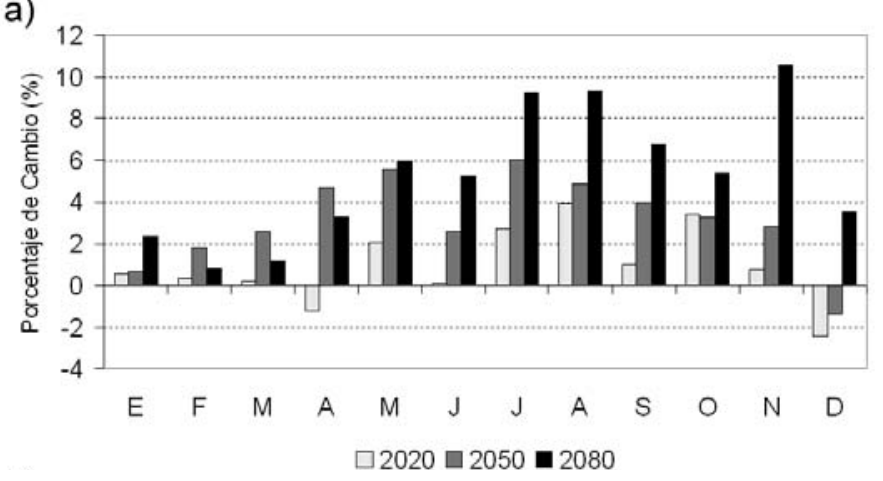

medida la responsabilidad de la variabilidad climática sobre la variabilidad en el rendimiento de la pastura en Salto para los años estudiados.

Existió concordancia con los resultados obtenidos de las regresiones simples: las variables indicadoras de humedad se encontraron entre las variables estimadoras del crecimiento de la pastura en verano y otoño, mostrando el efecto de la falta del recurso (deficiencia hídrica) (asociación directa con los totales y la frecuencia de precipitación e inversa con la frecuencia de días con precipitación inferior a la ETP). Para los suelos profundos, en el verano se agregaron la Tmín y la frecuencia de días con Tmáx superior a los $30^{\circ} \mathrm{C}$, y en el otoño, la frecuencia de heladas y la frecuencia de días con Tmáx superior a los $35^{\circ} \mathrm{C}$

En primavera se hizo presente el efecto negativo del monto de precipitaciones y frecuencia de días con precipitación inferior a la ETP para el suelo rojo y sólo el monto para el suelo negro. Dichas asociaciones no se presentaron para el suelo profundo durante esta época del año, confirmándose estadísticamente la tendencia esbozada en el análisis de correlación simple: el agua se convierte en limitante para el crecimiento de la pastura sólo en los suelos superficiales en primavera. Los suelos superficiales mostraron también su vínculo con la Tmáx, sugiriendo nuevamente la relación entre temperatura y demanda hídrica (ETP), indicador que no resultó significativo para los suelos profundos que cuentan con mayor disponibilidad de agua en esta estación del año. En los tres tipos de suelo se manifestó el efecto de las bajas temperaturas a través de la Tmín y la frecuencia de días con Tmín inferiores a los $10^{\circ} \mathrm{C}$.

En la Figura 6 se presentan algunos ejemplos de las curvas observadas y las estimadas a través de los modelos estadísticos encontrados. Las variabilidades interanuales de las tasas de crecimiento observados y estimados a partir de los modelos estuvieron en fase, y los años extremos en el rendimiento de la pastura se encontraron bien representados por la regresión.

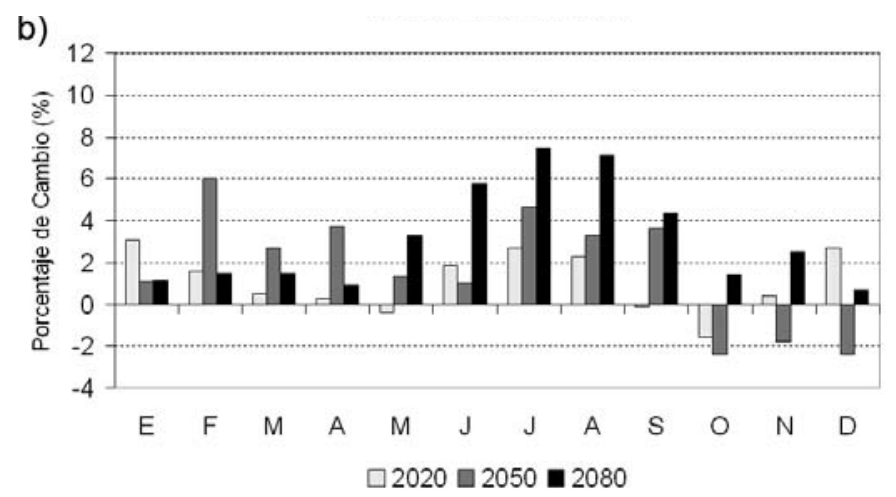

Figura 7 - Porcentaje de cambio de la precipitación mensual en Salto con respecto al período 1961-1990 para los escenarios A2 (a) y B2 (b). 
Cabe destacar aquí que el rendimiento de la pastura utilizado es un valor integrador que resume de forma simplificada complejas relaciones del sistema suelo-plantaatmósfera. A pesar de ello fue posible encontrar un conjunto de variables climáticas que expliquen un alto porcentaje de la variabilidad de la tasa de crecimiento de la pastura.

\subsection{Contextos Climáticos Futuros}

Los resultados de los ensambles de las proyecciones de anomalías mensuales de temperatura máxima, mínima y precipitación respecto a los valores observados en el período de referencia 1961-1990 fueron consistentes con otros estudios desarrollados para la región (Caffera et al., 2005). Las temperaturas máximas y mínimas presentaron incrementos sostenidamente, siendo los mismos mayores para el escenario A2 en la mayoría de los casos. Los mayores aumentos en Tmáx se obtuvieron entre los meses de octubre y febrero $\left(0,4-1,9^{\circ} \mathrm{C}\right)$ y en Tmín se verificaron entre septiembre y abril $\left(0,6\right.$ a $\left.2,5^{\circ} \mathrm{C}\right)$ (resultados no mostrados).

En general se presentarían incrementos en el porcentaje de precipitación mensual, con los mayores valores para 2080 en el escenario A2, entre 5 - 10\% (Figura 7 a). Los incrementos proyectados resultarían siempre superiores en el escenario A2 e incluso se presentarían decrementos en el escenario B2 para 2050 entre los meses de octubre y diciembre (Figura 7 b).

Los incrementos en la temperatura máxima promedio no alcanzarían el umbral térmico de sobrevivencia de las especies presentes (Tabla 1), aunque sí se desplazarían por encima de los umbrales óptimos. Las temperaturas mínimas promedio también mostrarían el mayor incremento en la época cálida (septiembre a abril), reforzando la proyección de un incremento generalizado de la temperatura para la secuencia primavera-verano-otoño que coincide con el ciclo de crecimiento del grupo C4 tropical de especies. Esta tendencia para la zona estudiada, coincide con lo reportado por Panario y Bidegain (1997) acerca de una esperable competencia ventajosa de las especies tropicales respecto a las templadas en escenarios de calentamiento y de continuidad del manejo tradicional del pastoreo en Uruguay. Sin embargo, al no incluirse la posible incidencia diferencial que el aumento de CO2 provocaría precisamente entre especies de distinto patrón de captación de $\mathrm{CO} 2$, las afirmaciones anteriores deberían considerarse preliminares hasta que puedan incorporarse dichos efectos en las mediciones de crecimiento y composición botánica del tapiz vegetal.

En cuanto a los posibles efectos de cambios en la precipitación, como se presentó en los ítems 3.1 y 3.2, no se detectaron limitantes hídricas para la pastura durante el invierno, por lo que aumentos en la oferta de agua no actuarían cubriendo una necesidad agroclimática en esta época del año. En primavera existieron limitantes de agua para la vegetación solamente en los suelos superficiales por lo que mayor precipitación podría mejorar la situación, no esperándose mayores consecuencias para los suelos profundos. Las proyecciones para el verano en general no se tradujeron en mayores aumentos de precipitación, siendo esta época muy crítica desde el punto de vista hídrico. Incluso aparecieron tendencias de menor lluvia en diciembre para los horizontes temporales de 2020 y 2050 (Figura 7 a y b). Considerando además el aumento proyectado en temperatura para esta época, podrían esperarse mayores deficiencias hídricas debido a los aumentos en la ETP. Según el horizonte temporal y el escenario considerado, los aumentos de precipitación en otoño se presentarían o no, en magnitudes relevantes (Figura 7). En este sentido, los incrementos proyectados desde febrero a abril para 2050 en el escenario B2 y desde abril en el A2, podrían ser interpretados como un posible efecto positivo, aunque esta tendencia no se verificó para 2020 ni 2080 (Figura 7).

\section{CONCLUSIONES}

De acuerdo al análisis de los valores observados de crecimiento de pastura natural en Salto (Uruguay) en el período 1980-1994, de los valores característicos de temperaturas medias y extremas y de la variabilidad interanual de las mismas, se confirmó la adecuación de los umbrales térmicos bioclimáticos propuestos por Baradas (1994) tomados como referencia. En este sentido, las categorías correspondientes a grupos vegetales tropicales (C4) adaptados a menores temperaturas y templados (C3) adaptados a mayores temperaturas, resultaron descriptivamente adecuadas para evaluar el crecimiento vegetal del campo natural de la región.

El análisis de variabilidad climática mostró resultados que no podrían haberse obtenido de las caracterizaciones y descripciones agroclimáticas actualmente disponibles. Esto permitió identificar índices climáticos que explicaron significativamente las variaciones en la disponibilidad estacional de forraje en distintos suelos, incorporando en forma paralela la variabilidad interanual del clima y la variabilidad interanual de crecimiento de pastura. Este análisis permitió cuantificar la elevada sensibilidad de la pastura a la oferta hídrica durante el otoño, que al analizarse en términos de su variabilidad se diferenció significativamente de la primavera como estación intermedia. Existieron aspectos que caracterizaron mejor el otoño, a saber, una mayor variabilidad interanual de la lluvia asociado al agotamiento del agua del suelo en la estación precedente. Este aspecto contribuye a explicar la disminución de la tasas de crecimiento de pastura natural en otoño, atribuido a la disminución de temperatura característica de esta época, que sin embargo a nivel medio es superior a la de primavera. 
Las proyecciones de temperatura en contextos de cambio climático brindan elementos para suponer mejores condiciones de competencia para las especies tropicales respecto a las especies templadas. Debido a que los mayores aumentos de temperatura se verificarían en el semestre más cálido del año, las especies tropicales tendrían condiciones favorables desde más temprano y durante mayor tiempo respecto a la situación histórica. En cuanto a las precipitaciones, los incrementos no cambiarían sustancialmente la situación respecto a lo histórico según las magnitudes proyectadas para primavera. En verano, la magnitud de los incrementos proyectados no parece relevante si se compara con el incremento previsto de temperatura y el aumento en la demanda hídrica atmosférica que ésta provoca. En invierno podrían existir efectos negativos por exceso hídrico y en otoño los resultados no se mostraron consistentes, por lo que no hay indicio aún para una posible tendencia.

Los resultados presentados fueron obtenidos con información climática y de crecimiento de pastura que abarcaron períodos de al menos 15 años, sin embargo resulta oportuno destacar que las proyecciones de largo plazo resultan más robustas cuanto mayor es el período histórico de referencia considerado. Por esto, los resultados presentados constituyen una evaluación preliminar del futuro contexto climático para el desarrollo de la pastura en la región noroeste de Uruguay.

\section{AGRADECIMIENTOS}

Los autores desean agradecer a los revisores anónimos cuyos comentarios y sugerencias mejoraron la calidad del trabajo. También agradecen los aportes académicos realizados por el Ing. Agr. Walter Baethgen y el Lic. Met. Mario Bidegain, al Instituto Interamericano para la Investigación del Cambio Global (IAI), el financiamiento de este trabajo a través del proyecto TISG-P-3 y a la Universidad de Buenos Aires a través del proyecto UBA X605.

\section{REFERENCIAS BIBLIOGRÁFICAS}

ALTESOR, A.; DI LANDRO, E.; MAY, H.; ESCURRA, E. Long term species change in a Uruguayan grassland. Journal of Vegetation Science, v. 9, p. 173-180, 1998.

BAETHGEN, W. Vulnerability of the agricultural sector of Latin America to Climate Change. Climate Research, v. 9, p. 1-7, 1997.

BARADAS, M. W. Crops requirements of tropical crops. In: Handbook of agricultural meteorology. New York, USA: J. F. Griffits. Oxford University Press, 1994. 189-202 p. BARROS, V.; MENÉNDEZ, A.; NAGY, G. El cambio climático en el Río de la Plata. Buenos Aires: Consejo Nacional de Investigaciones Científicas y Técnicas, 2005. 200 p.

BERRETA, E. J.Principales características climáticas y edáficas de la región de Basalto en Uruguay. In: Seminario de actualización de tecnologías en Basalto. Uruguay: Instituto Nacional de Investigación Agropecuaria, Serie técnica No 102. 1998.

BIDEGAIN, M.; CAFFERA, R.M. Comportamiento de la precipitación en la región sureste de América del Sur (Uruguay), asociado con los Índices de Oscilación Sur (IOS) y El Niño (TSM). In: CONGRESO ARGENTINO DE METEOROLOGIA, VI, 1991, Buenos Aires. Anais... Buenos Aires: CAM, 1991.

BIDEGAIN, M.; KRECL, P. Comportamiento de la temperatura en el Sudeste de Sudamérica (Uruguay), asociado al fenómeno ENSO. In: CONGRESO LATINOAMERICANO E IBERICO DE METEOROLOGIA, VIII, Y CONGRESO BRASILEÑO DE METEOROLOGIA, X, 1998, Brasilia. Anais... Brasilia: SBMET, 1998.

CAFFERA, R. M.; NAGY, G.; BIDEGAIN, M. Análisis de la estadística climática y desarrollo y evaluación de escenarios climáticos e hidrológicos de las principales cuencas hidrográficas del Uruguay y de su zona costera. Reporte Oficial de la Unidad de Cambio Climático, Dirección Nacional de Medio Ambiente. Montevideo: Ministerio de Vivienda, Ordenamiento Territorial y Medio Ambiente de Uruguay, 2005. $84 \mathrm{p}$.

CHEBATAROFF, J. Algunos aspectos evolutivos de la vegetación de la Provincia Fitogeográfica Uruguayense. Apartado de Revista Nacional. Montevideo, n. 201, p. 3-18, 1960.

CHIARA, J.P.; CRUZ, G. Preliminary results from the implementation of the SPUR2 model in Uruguay. Climate Research, v. 9, p. 9-15, 1997.

DEVORE, J. Probabilidad y estadística para ingeniería y ciencias. México: Thomson, 2005. 794 p.

DRAPER, N.; SMITH, R. Applied Regression Analysis. Second Edition, New York: John Wiley \& Sons, 1981.

DOMÍNGUEZ, A.; PRIETO, R. Perfil Ambiental del Uruguay 2002. Montevideo: Nordan Ed., 2002. 203p.

FERNÁNDEZ, C.J. Estimaciones de la densidad aparente, retención de agua disponible en el suelo a partir de la composición granulométrica y porcentaje de materia orgánica. In: REUNIÓN TÉCNICA DE LAFACULTAD DE AGRONOMÍA, 2a , 1979, Montevideo. Anais... Universidad de la República, 1979. 53p.

FOOD AND AGRICULTURE ORGANIZATION of the United Nations FAO. Método para la aplicación práctica de la fórmula de Penman para el cálculo de la evapotranspiración potencial. Serie Riego y Drenaje No 17. 1979.

GAY, C., C. CONDE, H. EAKIN, G. SANCHEZ TORRES, A. TEJEDA, R. SEILER, M. VINOCUR, A. GEYMONAT 
y M. WEHBE. Vulnerability and Adaptation to Climate Variability and Change: The Case of Farmers in Mexico and Argentina. Final Report Submitted to Assessments of Impacts and Adaptations to Climate Change (AIACC), Project No. LA29. Washington, DC: The International START Secretariat, 2006. 153p. http://www.aiaccproject. org/Final\%20Reports/final_reports.html

GIMÉNEZ, A. Climate change/variability in the mixed crop livestock production systems of the Argentinean, Brazilian and Uruguayan Pampas: climate scenarios, impacts and adaptive measures. Final Report Submitted to Assessments of Impacts and Adaptations to Climate Change (AIACC), Project No. LA27. Washington, DC: The International START Secretariat, 2006. 70p. http://www.aiaccproject. org/Final\%20Reports/final_reports.html

INTERGOVERNANMENTAL PANEL ON CLIMATE CHANGE IPCC. Working Group III. Summary for Policymakers. Climate Change: 2001: Mitigation. Cambridge UK: Cambridge University Press, 2001. 14p.

INTERGOVERNANMENTAL PANEL ON CLIMATE CHANGE IPCC, Working Group I. Climate Change 2007. The Physical Science Basis. 2007. Cambridge, UK and New York, USA: Cambridge University Press, 2007. 989p.

MINISTERIO DE GANADERÍA, AGRICULTURA Y PESCA, MGAP. Censo General Agropecuario 2000. In: Uruguay, Montevideo, 2002. CD-ROM1 y CD-ROM2.

MINISTERIO DE GANADERÍA, AGRICULTURA Y PESCA, MGAP. Carta de Suelos del Uruguay. In: Uruguay, Montevideo, 2005. 1CD-ROM.
NAKICENOVIC, N.; ALCAMO, J.; DAVIS, G.; DE VRIES, B.; FENHANN, J.; GAFFIN, S.; GREGORY, K.; GRÜBLER, A.; JUNG, T.Y.; KRAM, T.; LA ROVERE, E.L.; MICHAELIS, L.; MORI, S.; MORITA, T.; PEPPER, W.; PITCHER, H.; PRICE, L.; RAIHI, K.; ROEHRL, A.; ROGNER, H.-H.; SANKOVSKI, A.; SCHLESINGER, M.; SHUKLA, P.; SMITH, S.; SWART, R.; VAN ROOIJEN, S.; VICTOR, N.; DADI, Z.. Emissions Scenarios. A Special Report of Working Group III of the Intergovernmental Panel on Climate Change. Cambridge, UK and New York, USA: Cambridge University Press, 2000. 570p.

PANARIO, D.; BIDEGAIN, M. Climate change effects on grasslands in Uruguay. Climate Research, v. 9, p. 37-40, 1997.

RUSTICUCCI, M; RENOM, M. Variability and trends in indices of quality-controlled daily temperature extremes in Uruguay. International Journal of Climatology, v. 28, p. 1083-1095, 2008.

THORNTHWAITE, C.W.; MATHER, J.R. Instrucciones y tablas para el cómputo de la ETP y el balance hídrico. Buenos Aires: Instituto Nacional de Tecnología Agropecuaria, 1967. 55p.

WILBY, L.R.; DAWSON, C.W. Using SDSM Version 3.1 - A decision support tool for the assessment of regional climate change impacts. Climate Change Unit, Environment Agency of England and Wales; Department of Computer Science. UK: Loughborough University, 2004. 